Revue internationale P.M.E.

\title{
Les dirigeants de PME et la communication financière sur \\ l'Internet
}

\section{SME managers and Internet financial communication Los dirigentes de PyME y la comunicación financiera en internet}

\author{
Laetitia Pozniak et François Guillemette
}

Volume 26, numéro 3-4, 2013

URI : https://id.erudit.org/iderudit/1024522ar

DOI : https://doi.org/10.7202/1024522ar

Aller au sommaire du numéro

Éditeur(s)

Editions EMS - In Quarto SARL

ISSN

0776-5436 (imprimé)

1918-9699 (numérique)

Découvrir la revue

Citer cet article

Pozniak, L. \& Guillemette, F. (2013). Les dirigeants de PME et la communication financière sur l'Internet. Revue internationale P.M.E., 26(3-4), 139-167. https://doi.org/10.7202/1024522ar

\section{Résumé de l'article}

Cette recherche vise à étudier les relations entre les informations à caractère financier, que l'on peut retrouver sur le site internet d'une PME, et les éléments, favorables ou non à la communication financière, mis en avant par son dirigeant.

Pour appréhender cette problématique, des entretiens semi-directifs ont été menés auprès de dix dirigeants de PME cotées sur le Marché Libre de Bruxelles. Ce marché boursier fait partie d'Euronext New York Stock Exchange. C'est un marché non réglementé où les règles d'admission à la cote et les obligations ont été allégées pour permettre aux PME de faire et de lever des capitaux sans se soumettre aux règles contraignantes d'un marché réglementé. Les sites internet des dix entreprises de notre corpus ont également été analysés.

L'analyse des sites internet permet de souligner l'effort volontaire de communication financière réalisé par les dirigeants de PME. Il ressort des entretiens avec ces dirigeants que plusieurs éléments sont mis dans la balance par les dirigeants lorsqu'ils pèsent le pour et le contre de la diffusion d'informations financières sur le site internet de leur PME. L'élément qui ressort en particulier, notamment à cause de son caractère favorable à la communication, est le respect des investisseurs. En effet, nous pouvons établir clairement un lien entre les dirigeants qui mettent un point d'honneur à respecter leurs investisseurs et le fait que leurs sites internet contiennent plus d'informations financières que les sites des autres dirigeants. Par contre, il ressort que la méfiance envers les concurrents constitue clairement un élément freinant la diffusion d'informations. En effet, les sites internet des dirigeants ayant évoqué la crainte de la concurrence contiennent nettement moins d'informations financières que les autres sites. Un lien est donc établi entre les raisons avancées par le dirigeant et le contenu à caractère financier présent sur le site internet de sa PME. 


\title{
Les dirigeants de PME et la communication financiere sur l'Internet ${ }^{1}$
}

\begin{abstract}
Laetitia POZNIAK ${ }^{2}$
Laetitia PozNiak est docteure en sciences économiques et de gestion, et assistante au sein du service finance de la Faculté Warocqué d'économie et de gestion de l'UMONS en Belgique. Elle enseigne les travaux pratiques relatifs au cours d'introduction à l'analyse financière, éléments de mathématiques financières, marchés boursiers et gestion de portefeuille. Ses domaines de recherche sont la communication financière, les marchés boursiers non réglementés, les PME et l'Internet.
\end{abstract}

Faculté Warocqué déconomie et de gestion de l'UMONS 20, place Warocqué 7000 MONS, Belgique laetitia.pozniak@umons.ac.be

\section{François GUILLEMETTE}

François Guillemette (Ph. D. en éducation, Université du Québec, 2005 ; Ph. D. en théologie, Université Laval, 1993) est professeur au Département des sciences de l'éducation à l'Université du Québec à Trois-Rivières et adjoint pédagogique au vice-décanat du Campus Mauricie de la Faculté de médecine de l'Université de Montréal. Il est également consultant-chercheur pour l'ACDI, spécifiquement dans l'approche par compétences en enseignement supérieur. Il enseigne la méthodologie de la recherche et donne des ateliers en recherche qualitative au Canada et en Europe francophone.

Université du Québec à Trois-Rivières 3351, boul. des Forges

CP 500

TROIS-RIVIÈRES, Québec guillefr@uqtr.ca

\section{RÉSUMÉ}

Cette recherche vise à étudier les relations entre les informations à caractère financier, que l'on peut retrouver sur le site internet d'une PME, et les éléments, favorables ou non à la communication financière, mis en avant par son dirigeant.

1 Le terme Internet est utilisé avec un « $\mathrm{i}$ » minuscule lorsqu'il s'agit de l'adjectif et avec un «I » majuscule lorsqu'il s'agit du nom propre, et ce en accord avec $:<$ http://www.insolus.com/articleinternet-majuscule-minuscule\#axzz2GBOFbDWk>.

2 Laetitia Pozniak tient à remercier Christophe Lejeune, expert scientifique à l'ULG, pour ses conseils méthodologiques lors des débuts hésitants de cette recherche. 
Pour appréhender cette problématique, des entretiens semi-directifs ont été menés auprès de dix dirigeants de PME cotées sur le Marché Libre de Bruxelles. Ce marché boursier fait partie d'Euronext New York Stock Exchange. Cest un marché non réglementé où les règles d’admission à la cote et les obligations ont été allégées pour permettre aux PME de faire et de lever des capitaux sans se soumettre aux règles contraignantes d’un marché réglementé. Les sites internet des dix entreprises de notre corpus ont également été analysés.

L'analyse des sites internet permet de souligner l'effort volontaire de communication financière réalisé par les dirigeants de PME. Il ressort des entretiens avec ces dirigeants que plusieurs éléments sont mis dans la balance par les dirigeants lorsqu'ils pèsent le pour et le contre de la diffusion d'informations financières sur le site internet de leur PME. Lélément qui ressort en particulier, notamment à cause de son caractère favorable à la communication, est le respect des investisseurs. En effet, nous pouvons établir clairement un lien entre les dirigeants qui mettent un point d'honneur à respecter leurs investisseurs et le fait que leurs sites internet contiennent plus d'informations financières que les sites des autres dirigeants. Par contre, il ressort que la méfiance envers les concurrents constitue clairement un élément freinant la diffusion d'informations. En effet, les sites internet des dirigeants ayant évoqué la crainte de la concurrence contiennent nettement moins d'informations financières que les autres sites. Un lien est donc établi entre les raisons avancées par le dirigeant et le contenu à caractère financier présent sur le site internet de sa PME.

\section{MOTS CLÉS}

PME, Communication financière, Internet, NYSE Euronext, Marché Libre, Marchés boursiers non réglementés, Dirigeant/propriétaire

\section{SME managers and Internet financial communication}

\section{SuMMARY}

This research focuses on studying the relations between internet financial communication of an SME and the arguments, favorable or not, to the financial disclosure, put forward by the manager.

To reach this goal, ten managers of SMEs led interviews quoted on the Free Market of Brussels. This stock market is part of Euronext New York Stock Exchange. It is an unregulated market where the rules of admission to quotation and the obligations were eased to allow SMEs to raise capital. The web sites of ten companies of our corpus were also analyzed.

Analyzing web sites highlights the SME managers' voluntary effort of financial communication.Interviews with these managers show that several elements are put in the balance when deciding to communicate or not. The element which stands out in favor of internet financial communication is the respect for the investors. Indeed, we can clearly establish a link between the managers who make it a point of honor to respect their investors and the fact that their web sites contain more financial information than the sites of the other managers. However, it emerges that the fear of competitors is an element that slows down the internet financial communication. Indeed, the web sites of the managers talking about the fear of the competitors clearly contain less financial information than the other sites. A link is thus established between the reasons put forward by the managers and the internet financial communication of their SMEs.

\section{KEY WORDS}

SME. Financial communication, Internet, NYSE Euronext, The Free Market, Not regulated financial markets. Managers 


\title{
Los dirigentes de PyME y la comunicación financiera en internet
}

\begin{abstract}
RESUMEN
Esta investigación tiene como objetivo estudiar la relación entre la información de carácter financiero que se puede encontrar en la página web de las PyMEs y los elementos, favorables o desfavorables de la comunicación financiera, destacados por su director.

Para alcanzar este objetivo, se relizaron entrevistas a 10 gerentes de PyMEs valoradas en el Libre mercado de Bruselas. Este mercado bursátil forma parte de Euronext New York Stock Exchange. Se trata de un mercado no regulado donde las reglas de admisión a la cotización y las obligaciones fueron aligeradas para permitir a las PyMEs la obtención de capital. Se analizaron también las páginas web de 10 empresas.

El análisis de páginas web permite subrayar el esfuerzo voluntario de comunicación financiera realizada por los gerentes de las PyMEs. Las entrevistas con estos gerentes muestran que varios elementos son valorados cuando ellos consideran los pros y los contras de la difusión de la información financiera en sus páginas web. El elemento que se destaca a favor de la comunicación financiera de Internet es el respeto a los inversores. De hecho, podemos observar que los gerentes que se comprometen a respetar a sus inversores ofrecen más información financiera en sus páginas web que aquellos gerentes que no muestran la misma actitud. Por otra parte, el miedo a los competidores es un elemento que reduce la velocidad de la comunicación financiera de Internet. Los sitios web de los gerentes preocupados por los competidores contienen información claramente menos financiera que el resto de sitios web. Se puede establecer una relación entre los motivos avanzados por los gerentes y la comunicación financiera presente en las páginas web de las PyMEs.
\end{abstract}

\section{Palabras claves}

PyME, Comunicación Financiera, Internet, Bolsa de Nueva York, El Libre mercado, Mercados no regulados, Gerentes

\section{Introduction}

Entre 2000 et 2009, le nombre d'usagers d'Internet à travers le monde a crû de près de $400 \%$, pour atteindre plus d'un milliard 800 millions de personnes fin 2009, ce qui représente un peu plus du quart de la population mondiale. En Europe, plus de la moitié de la population utilise l'Internet ${ }^{3}$. Ces statistiques reflètent la place très importante de l'outil internet dans la vie quotidienne des gens. Par exemple, une étude réalisée par Fleishmann-Hillard et Havas Interactive en $2008^{4}$ a révélé que les décisions des consommateurs étaient plus influencées par l'Internet que par les médias traditionnels.

$3<$ http://www.internetworldstats.com/stats.htm >.

4 Westphalen et Libaert, 2009, 240 p. 
Le monde de la finance n'est pas en reste. Selon une étude TNS Sofres réalisée en 2006, $90 \%$ des actionnaires étaient internautes et $50 \%$ consultaient les sites des sociétés cotées. Léger (2008) affirme que l'Internet permet de développer la Shareholding Relationship Management et offre de nouvelles possibilités à l'entreprise pour entretenir sa relation avec l'actionnaire tels que l'information via $e$-mailing, la retransmission de réunions et les assemblées sur le site internet, le contenu d'un site corporatif, etc.

De nombreuses études se sont penchées sur l'utilisation de l'Internet comme vecteur de communication vers les investisseurs et partenaires financiers. Certains chercheurs ont tenté de faire ressortir les déterminants du degré de communication financière sur le site internet de l'entreprise (Ashbaugh, Johnstone et Warfield, 1999 ; Bonson et Escobar, 2002 ; Debreceny, Gray et Rahman, 2002 ; Mendes-da-Silva et Christensen, 2004 ; Almilia, 2009 ; Pozniak, 2010). Ces études ont pour objet de recherche la grande entreprise cotée sur des marchés boursiers réglementés. Or, notre recherche porte sur des petites et moyennes entreprises (PME) cotées sur le marché non réglementé belge. Les PME jouent un rôle très important dans l'économie de leur pays (Sutanonpaiboon et Pearson, 2006). En Belgique, elles représentent 99,8 \% des entreprises, elles emploient 66,6\% des travailleurs et elles contribuent à hauteur de 57,4 \% de la valeur ajoutée (European Commission, 2008, données 2005). Les caractéristiques particulières des PME ont été observées par plusieurs chercheurs (Torrès, 2007a, b ; Filion, 2007a, b ; St-Pierre, 1999). Raymond et al. (1990), ont étudié le lien entre ces spécificités des PME et l'implantation des technologies de l'information. Notre problématique de recherche constitue un angle spécifique détude de ce lien, en ce sens que nous avons voulu comprendre, dans le contexte d'un type particulier de PME, le lien entre les raisons évoquées par leurs dirigeants pour l'utilisation de l'Internet et la diffusion des informations financières sur les sites internet de ces PME.

Loriginalité de notre recherche réside notamment dans son intention d'explorer un terrain vierge d'expérimentation. À notre connaissance, aucune recherche scientifique (excepté Pozniak, 2010) ne traite du cas des entreprises cotées sur les marchés boursiers non réglementés où évoluent essentiellement des PME. Nous sommes donc en présence de ce que Bouchard (2000) appelle un «territoire inexploré » ou encore ce que Deleuze ${ }^{6}$ qualifie d' " oubli de la science ». En effet, notre étude s'intéresse aux PME cotées sur le marché non réglementé et non organisé de la Bourse de Bruxelles ${ }^{7}$ : le Marché Libre. Créé en novembre 2004 sur la base du modèle parisien existant depuis 1996, le Marché Libre permet aux entreprises de lever des capitaux sans avoir à satisfaire l'ensemble des contraintes qui régissent un marché réglementé.

Ce marché, composé exclusivement de PME (au regard de la définition européenne), a pour principale caractéristique de n'imposer aux entreprises cotées aucune obligation en matière de communication financière. Dès lors, la diffusion d'informations financières par ces entreprises est laissée à la discrétion du dirigeant, tant au niveau du contenu, que du choix des vecteurs de communication. L'existence d'une telle communication relève donc

5 Westphalen et Libaert, 2009, 337 p.

6 Villani, 1996.

7 La Bourse de Bruxelles appartient au groupe NYSE Euronext. 
d'un effort volontaire de la part du dirigeant. En effet, comme le précise Pourtier (2004), « en délimitant ce qui est obligatoire, nous soulignerons ce qui est, par défaut, volontaire». En l'occurrence, sur le Marché Libre de Bruxelles, aucune diffusion d'informations financières n'est requise, toute communication financière peut donc être qualifiée de volontaire ; de là la pertinence deétudier le lien entre la «volonté » communicationnelle des dirigeants et la diffusion elle-même.

L'Internet représente à l'heure actuelle un vecteur incontournable de communication. Il permet aux PME de communiquer avec leurs parties prenantes, tout comme les grandes entreprises et ce, malgré leurs moyens limités (Amarasena, 2008). Il est à noter que, sur le marché réglementé de Bruxelles (Euronext), la création d'un site internet et la diffusion d'informations financières sur ce vecteur sont requises par l'Autorité des services et marchés financiers $^{8}$ (FSMA, 2012). Ce n'est bien entendu pas le cas sur le Marché Libre. Dans un tel contexte, où, d'une part, aucune contrainte de communication n'est imposée aux PME cotées sur le Marché Libre et où, d'autre part, l'Internet offre la possibilité aux PME de communiquer facilement, rapidement et à moindres frais, il est intéressant détudier les choix de communication posés librement par les dirigeants de ces PME et les raisons invoquées.

Cette recherche tente, dans un premier temps, de relever les informations financières que les dirigeants de PME diffusent sur leur site internet. Dans un second temps, elle cherche à comprendre ce qui a amené ces dirigeants à publier ou non des informations financières sur leur site internet. Enfin, nous tentons détablir des relations entre les deux premiers types d'informations recueillies, c'est-à-dire des relations entre les informations financières diffusées sur le site internet et les intentions évoquées par les dirigeants. Afin d'appréhender ces questions, dix dirigeants de PME du Marché Libre de Bruxelles ont été interviewés et le contenu de leurs sites internet respectifs a été analysé.

Cet article est divisé en quatre parties. Tout d'abord, une section présente notre cadre conceptuel et est donc consacrée à la communication financière sur l'Internet, aux spécificités de la PME, ainsi qu’à la place prépondérante du dirigeant en son sein. Dans la deuxième partie, la section "méthodologie ", nous présentons le processus de constitution du corpus de données, de même que les procédures d'analyse. En troisième lieu, nous exposons les résultats de notre recherche et les discutons. Enfin, la conclusion présente l'essentiel de nos découvertes, les limites de notre étude, ainsi que les pistes de recherche à explorer.

\section{CADRE CONCEPTUEL}

Le cadre conceptuel est constitué de trois éléments. Les deux premiers éléments sont consacrés à la définition de la PME et à la spécificité de celle-ci. Le troisième est le fruit d'une recension des écrits scientifiques relatifs aux recommandations en termes de communication financière sur l'Internet et relatifs aux déterminants du niveau de diffusion d'informations financières sur l'Internet.

8 En abrégé FSMA pour 'Financial Services and Markets Authority'. 


\subsection{La PME : définition}

Comme le fait remarquer Marchesnay (1991, p. 11), "il n'y a pas "la" PME»; il existe une grande diversité de types de PME. Les définitions quantitatives de la PME sont relativement différentes d'un pays à l'autre. Par exemple, pour des pays tels que la France, l'Allemagne, les États-Unis et le Japon, une entreprise est considérée comme une PME dès qu'elle n’emploie pas plus de 500 travailleurs (Filion, 2007a). Dans le cadre de cette recherche, nous utiliserons la définition européenne étant donné que les PME étudiées sont cotées sur le Marché Libre de Bruxelles, qui fait partie, depuis 2000, de la place boursière européenne : Euronext ${ }^{9}$. La définition européenne stipule que "la catégorie des PME est constituée des entreprises qui occupent moins de 250 personnes et dont le chiffre d'affaires annuel n'excède pas 50 millions d'euros ou dont le total du bilan n'excède pas 43 millions d'euros" (Commission européenne, 2006 , p. 5). Elle précise également que sont considérées comme " petites » les entreprises qui emploient moins de 50 personnes et dont le chiffre d'affaires annuel ou le total du bilan annuel ne dépasse pas 10 millions d'euros. On parlera de " micro-entreprise " dès lors, que moins de 10 personnes sont employées et que le chiffre d'affaires annuel et le total du bilan annuel n'excèdent pas 2 millions d'euros.

Outre les critères quantitatifs, Julien (1997) prend en considération des éléments qualitatifs pour définir la PME, à savoir une petite taille, la centralisation de la gestion autour du dirigeant, une faible spécialisation (de la direction et du personnel) dans la répartition des tâches, des systèmes d'information interne et externe simples ou peu organisés, ainsi qu'une stratégie intuitive ou peu formalisée.

Ces caractéristiques particulières de la PME seront abordées au point suivant et leur incidence éventuelle sur la communication financière sur l'Internet sera présentée dans la partie consacrée aux résultats.

\subsection{Les spécificités de la PME}

Selon St-Pierre (1999), il est essentiel de prendre en compte les spécificités de la PME et de ne pas la considérer comme une future grande entreprise. De très nombreux chercheurs se sont penchés sur les spécificités des PME, entre autres, Torrès, 2007a, b ; Filion, 2007a, b et St-Pierre, 1999. L'impact de ces spécificités sur le développement, l'exploitation et l'utilisation de l'informatique a été étudié par Raymond et al. (1990). Nous soulignons ici les caractéristiques qui seraient susceptibles d'influencer la communication financière sur l'Internet.

En termes de ressources humaines, les PME sont caractérisées par une petite équipe dirigeante et le manque de personne spécialisée dans les technologies d'informations et de communication (Sutanonpaiboon et Pearson, 2006). Il n'y a que rarement des directions de la communication chez les PME ou du personnel attitré exclusivement à la communication. Le cas échéant, ces entreprises font plutôt appel à des conseillers externes pour ce travail spécialisé (Westphalen et Libaert, 2009). Ce manque de ressources humaines dédiées

9 Euronext ayant fusionné avec le NYSE Group en 2007, pour créer le NYSE Euronext. 
à la communication dans les PME constitue un problème important selon Raymond et al. (1990). En effet, dans ces structures de taille réduite, aux moyens limités, le niveau de diffusion d'informations financières sur l'Internet sera bien inférieur à celui des grandes entreprises et les conséquences de cette pauvreté de moyens communicationnels sont évidentes.

Au sein des PME, on retrouve un système d'information de proximité (Torrès, 2007a), c'està-dire une communication interne simple, peu structurée et basée essentiellement sur l'oral. C'est ce genre de système sous-développé qui est privilégié par le propriétaire-dirigeant.

Ces deux caractéristiques (le manque de ressources dédiées à la communication et les systèmes sous-développés) reflètent le manque de moyens humains ou technologiques dont souffrent les PME et qui affecte sans doute leur communication financière sur l'Internet.

Plusieurs études se sont penchées sur les déterminants de l'adoption du e-commerce dans les PME. L'enthousiasme et la perception du dirigeant/propriétaire sont l'un des facteurs clés mis en évidence par ces recherches (Sutanonpaiboon et Pearson, 2006 ; Pearson et Grandon, 2005 ; Grandon et Pearson, 2004 ; Lertwongsatien et Wongpinunwatana, 2003 ; Wong, 2003 ; Mirchandani et Motwani, 2001). Mehrtens, Cragg et Mills (2001) affirment que le dirigeant/propriétaire de PME a souvent un rôle significatif dans l'adoption de nouvelles technologies de l'information. Pour développer une stratégie internet, une PME a besoin d'un dirigeant qui reconnaisse le potentiel d'Internet et les opportunités qu'il représente pour l'entreprise. Quant au succès du site internet de l'entreprise, Vescovi (2000) souligne qu'il dépend de la présence d'un "champion », c'est-à-dire d'une personne très influente dans l'entreprise qui cernera la valeur stratégique de l'Internet et qui portera le leadership du projet. Comme Vescovi le rappelle, ce leader ne peut que rarement être une autre personne que le dirigeant dans une PME.

Dans le même ordre d'idées, Filion (2007b) signale qu'on ne peut parler de PME sans aborder la personnalisation de la direction et de la gestion de l'entreprise. La proximité et l'omniprésence du dirigeant impliquent que sa personnalité influence grandement ce qui se passe dans l'entreprise. Dans cette perspective, Werner (2008) étudie en particulier l'influence de l'identité chrétienne du dirigeant sur la manière dont il gère l'entreprise. L'auteur se penche sur les dirigeants/propriétaires de PME partant du postulat qu'ils occupent une position idéale pour partager leurs valeurs personnelles dans l'entreprise. De Zoysa et Herath (2007) montrent également le pouvoir et l'influence considérable du propriétaire-dirigeant de PME. Raymond et al. (1990), de même que St-Pierre (1999), abondent dans ce sens et établissent que les comportements et prises de décisions dans la PME sont fortement attachés à l'expérience, à l'intuition et aux motivations personnelles de l'entrepreneur. D’autres recherches ont démontré le lien entre la personnalité du dirigeant/propriétaire de PME et la performance de celle-ci (De Zoysa et Herath, 2007 ; Kotey et Meredith, 1997 ; Wijewardena et Cooray, 1996 ; Davidsson, 1991 ; Ibrahim et Goodwin, 1986 ; Chaganti et Chaganti, 1983).

Les comportements particuliers des dirigeants de PME ont été expliqués par Torrès (2007a) en utilisant la notion de proximité. Cette notion est, selon lui, «l'élément central qui permet non seulement de décrire une grande diversité de formes de PME, mais aussi d'expliquer en grande partie les comportements particuliers des dirigeants de PME»(Torrès, 2007a, p. 24). Mentionnons à cet égard la notion de proximité hiérarchique selon laquelle le pouvoir est centralisé entre les mains du propriétaire-dirigeant, et ce, grâce à une structure relative- 
ment compacte, ainsi que la notion de proximité fonctionnelle qui désigne le fait que le propriétaire-dirigeant assure souvent plusieurs fonctions dans l'entreprise.

Tous ces éléments mettent en lumière la place prépondérante du dirigeant dans sa PME et son influence à plusieurs niveaux. Nous verrons plus loin en quoi cette place prépondérante du propriétaire-dirigeant a un impact sur la communication financière par le site internet de sa PME.

Selon Torrès (2007a), il existe au sein des PME une finance de proximité, c'est-à-dire que le capital de l'entreprise est fortement concentré entre les mains du dirigeant dont le patrimoine est fusionné avec celui de l'entreprise. Cela se vérifie pour notre recherche qui porte sur les PME cotées sur le Marché Libre de Bruxelles dont la structure d'actionnariat est caractérisée par un faible pourcentage des titres dans le public (en moyenne $18 \%$ ).

Le lien entre la structure de propriété et la communication financière a été abordé dans plusieurs recherches. Selon Labelle et Schatt (2005), en fonction de la structure financière de l'entreprise, la communication financière émanant des dirigeants présente deux gains potentiels. D'une part, lorsque l'actionnariat est très dilué, la communication financière permet de réduire les conflits d'intérêts entre actionnaires et dirigeants. D’autre part, lorsque l'actionnariat est très concentré, la communication financière permet d'augmenter les volumes de transactions et ainsi la liquidité des titres.

Pour ce qui est des entreprises à capital dispersé, l'avantage lié à une communication financière de qualité fait référence à la théorie de l'agence, théorie introduite par Jensen et Meckling en 1976, et dans laquelle ils expliquent que la séparation de la propriété et de la gestion de l'entreprise implique des conflits entre actionnaires et dirigeants ainsi que des coûts d'agence. Ces coûts comprennent à la fois les coûts supportés par les actionnaires pour surveiller les dirigeants et pour s'assurer qu'ils maximisent la valeur de l'entreprise, de même que les coûts supportés par les dirigeants pour prouver leur bonne foi aux actionnaires et pour éviter de se faire remplacer (Labelle et Schatt, 2005). Le risque de coûts d'agence est d'autant plus élevé que le capital est dispersé (Abdelsalam, Bryant et Street, 2007). Plusieurs travaux ont démontré cette relation positive entre la proportion des parts détenues par le public et le degré de diffusion d'informations financières sur l'Internet (Asbaugh, Johnstone et Warfield, 1999 ; Ho et Wong, 2001 ; Bollen, Hassink et Bozic, 2006).

Quant aux entreprises à capital concentré, une plus grande diffusion d'informations financières permet de susciter l'intérêt des analystes et de réduire l'asymétrie d'information (Labelle et Schatt, 2005). Barredy et Darras (2008, p. 6) précisent que «lorsque le dirigeant possède une fraction significative de capital, les intérêts du dirigeant tendent à saligner avec ceux des actionnaires et la dépendance informationnelle de l'actionnaire minoritaire est réduite». Ces chercheures supposent alors que, dans les entreprises familiales, où par définition le capital est concentré aux mains d'une famille, les intérêts des actionnaires minoritaires sont largement protégés par la présence de la famille au sein de la direction et du capital de l'entreprise. Dès lors, l'incitation à la divulgation d'informations financières est réduite. Les mêmes chercheures démontrent aussi que les entreprises où la famille est fortement présente dans le capital diffusent peu d'informations financières.

Ben Ali et Gettler-Summa (2006), dans leur étude des entreprises françaises cotées faisant partie du SBF 120, arrivent également à cette conclusion : les entreprises contrôlées par des 
familles diffusent moins d'informations financières que les autres entreprises. Les familles participent activement à la gestion de l'entreprise et ont accès à toute l'information nécessaire. Ainsi, la demande de diffusion d'informations est quasi nulle et les manifestations de la communication financière demeurent sous-développées.

Dans notre recherche, la plupart des dirigeants rencontrés (X, Z, C, D et G) détiennent plus de $50 \%$ du capital de l'entreprise (voir Tableau 3). Il s'agit donc d'un actionnariat concentré aux mains du dirigeant/propriétaire. D’après Labelle et Schatt (2005), nous sommes donc dans le second cas de figure où la communication financière permettrait d'attirer l'attention des analystes, de réduire l'asymétrie d'information, d'augmenter les volumes de transactions et donc la liquidité.

Par contre, certaines entreprises de notre corpus ( $\mathrm{Y}, \mathrm{B}$ et F) présentent un actionnariat fortement dilué. Selon Labelle et Schatt (2005), elles seraient donc dans le premier cas de figure où la communication financière permettrait de réduire les conflits d'intérêts entre actionnaires et dirigeants. On s'attend donc à ce que ces entreprises communiquent davantage que les autres.

\subsection{La communication financière sur l'Internet}

La diffusion d'informations financières sur l'Internet a fait l'objet de plusieurs travaux de recherche (Allam et Lymer, 2003 ; Oyelere, Laswad et Fisher, 2003 ; Xiao, Yangb et Chow, 2004 ; Gowthorpe, 2004 ; Khadaroo, 2005 ; Lybaert, 2005 ; Pervan, 2006 ; Euronext, 2006 ; Dutta et Bose, 2007 ; Abdelsalam, Bryant et Street, 2007 ; Oyelere et Mohamed, 2007 ; Léger, 2008 ; Barredy et Darras, 2008 ; Gabteni, 2011). Ces travaux ont fait ressortir les éléments susceptibles d'intéresser les investisseurs et qui devraient figurer sur le site internet de l'entreprise. Khadaroo (2005) précise qu'outre les informations financières, les informations relatives à l'entreprise, ses parts de marchés et ses organes de gouvernance sont essentiels.

Au sein de la communauté scientifique, la disponibilité du rapport annuel apparait comme indispensable (Khadaroo, 2005 ; Lybaert, 2005 ; Pervan, 2006 ; Euronext, 2006 ; Dutta et Bose, 2007 ; Léger, 2008 ; Barredy et Darras, 2008) ainsi que les résultats intermédiaires (Dutta et Bose, 2007 ; Abdelsalam, Bryant et Street, 2007 ; Khadaroo, 2005 ; Lybaert, 2005 ; Xiao, Yangb et Chow, 2004 ; Gowthorpe, 2004) et, dans une moindre mesure, les rapports d'audit (Lybaert, 2005 ; Dutta et Bose, 2007). Il est également judicieux de présenter les ratios importants (Allam et Lymer, 2003 ; Xiao, Yangb et Chow, 2004 ; Lybaert, 2005 ; Dutta et Bose, 2007 ; Gabteni, 2011). La revue de presse (Allam et Lymer, 2003 ; Gowthorpe, 2004 ; Khadaroo, 2005 ; Lybaert, 2005 ; Pervan, 2006 ; Euronext, 2006 ; Dutta and Bose, 2007 ; Abdelsalam, Bryant et Street, 2007 ; Léger, 2008), l'organigramme de l'entreprise (Pervan, 2006 ; Euronext, 2006 ; Dutta and Bose, 2007 ; Gabteni, 2011) et le prospectus d'introduction en Bourse (Euronext, 2006 ; Léger, 2008) sont vivement recommandés.

De nombreux chercheurs (Allam et Lymer, 2003 ; Xiao, Yangb et Chow, 2004 ; Khadaroo, 2005 ; Lybaert, 2005 ; Pervan, 2006 ; Dutta et Bose, 2007 ; Léger, 2008 ; Barredy et Darras, 2008 ; Gabteni, 2011) affirment que l'historique du cours de l'action ainsi que les dividendes doivent être disponibles sur le site internet d'une entreprise cotée. D’autres (Khadaroo, 2005 ; Pervan, 2006 ; Barredy et Darras, 2008 ; FSMA, 2012) ajoutent qu'une re- 
lation particulière doit être nouée avec les investisseurs et cela peut se traduire par une page spéciale qui leur est dédiée ou par un autre moyen, tels un contact spécifique (par courriel ou par téléphone), un forum de discussion, le calendrier financier, la lettre aux actionnaires, le guide et les droits des actionnaires et la possibilité de s'abonner à une newsletter.

Plusieurs recherches ont eu pour ambition d'identifier les facteurs influençant la diffusion d'informations financières sur le site internet de l'entreprise (Ashbaugh, Johnstone et Warfield, 1999 ; Bonson et Escobar, 2002 ; Debreceny, Gray et Rahman, 2002 ; Mendesda-Silva et Christensen, 2004 ; Almilia, 2009 ; Pozniak, 2010). Une synthèse des variables explicatives du niveau de communication financière sur l'Internet, mises en lumière par ces travaux, est présentée dans le tableau 1.

TABLEAU 1. SYNTHÈSE DES DÉTERMINANTS DE LA COMMUNICATION FINANCIÈRE SUR L'INTERNET

\begin{tabular}{|c|c|c|c|c|}
\hline Auteurs & Pays & Échantillon & Variables & Effet observé \\
\hline \multirow{3}{*}{$\begin{array}{l}\text { Ashbaugh, Johnstone } \\
\text { et Warfield (1999) }\end{array}$} & \multirow{3}{*}{ États-Unis } & \multirow{3}{*}{290} & Taille & Positif \\
\hline & & & Performance & Positif \\
\hline & & & Dispersion capital & Positif \\
\hline \multirow{3}{*}{$\begin{array}{l}\text { Bonson } \\
\text { et Escobar (2002) }\end{array}$} & \multirow{3}{*}{ Europe } & \multirow{3}{*}{20} & Taille & Positif \\
\hline & & & Secteur & Positif \\
\hline & & & Pays d'origine & Positif \\
\hline \multirow{3}{*}{$\begin{array}{l}\text { Debreceny, Gray } \\
\text { et Rahman (2002) }\end{array}$} & \multirow{3}{*}{22 pays } & \multirow{3}{*}{660} & Taille & Positif \\
\hline & & & Secteur IT & Positif \\
\hline & & & Cotation & Positif \\
\hline \multirow{2}{*}{$\begin{array}{l}\text { Mendes-da-Silva } \\
\text { et Christensen (2004) }\end{array}$} & \multirow{2}{*}{ Brésil } & \multirow{2}{*}{291} & Taille & Positif \\
\hline & & & Performance & Négatif \\
\hline \multirow{2}{*}{ Almilia (2009) } & \multirow{2}{*}{ Indonésie } & \multirow{2}{*}{303} & Taille & Positif \\
\hline & & & Endettement & Positif \\
\hline \multirow{3}{*}{ Pozniak (2010) } & \multirow{3}{*}{ Belgique } & \multirow{3}{*}{37} & Secteur IT & Positif \\
\hline & & & Marché de cotation & Positif \\
\hline & & & Performance & Négatif \\
\hline
\end{tabular}

La taille de l'entreprise, son niveau d'endettement, sa structure financière, sa performance et son appartenance au secteur IT, notamment, se sont révélés être des facteurs influençant le degré de diffusion d'informations financières sur le site internet de l'entreprise. Notons que la grande majorité de ces travaux ont étudié les grandes entreprises (sauf Pozniak, 2010). Or, la présente recherche s'intéresse aux PME cotées sur un marché boursier non réglementé, le Marché Libre de Bruxelles. 


\section{MÉTHODOLOGIE}

Comme nous le disions plus haut, cette recherche poursuit trois objectifs :

- connaître les informations financières que les dirigeants de PME diffusent sur leur site internet ;

- comprendre ce qui a amené ces dirigeants à publier ou non des informations financières sur leur site internet;

- établir des relations entre les deux premiers types d'informations recueillies, c'est-à-dire des relations entre les informations financières diffusées sur le site internet et les intentions évoquées par les dirigeants.

Notre recherche s'inscrit dans une démarche qualitative, tout indiquée lorsque l'on est dans une démarche de compréhension d'un phénomène (Evrard, Pras et Roux, 2009 ; Paillé, 2007 ; Miles et Huberman, 2003). Plus précisément, nous cherchons à comprendre ce qui est vécu par les dirigeants des PME du Marché libre en ce qui concerne la diffusion des informations financières sur leur site internet. Dans la logique épistémologique de la recherche qualitative, on ne peut étudier un phénomène humain quà partir de la conscience qu’en ont les personnes qui le vivent (Blumer, 1969 ; Dilthey, 1942 ; Husserl, 1977 ; Schutz, 1987 ; Weber, 1949). Cette recherche est essentiellement interprétative parce que le vécu humain est précisément humain parce qu'il a un sens et donc parce qu'il est interprété (Blumer, 1969 ; Corbin et Strauss, 2008). Par ailleurs, on ne peut avoir accès à la conscience du vécu, et donc au sens ou à l'interprétation, que par le discours issu de la prise de conscience. Ainsi, la recherche qualitative privilégie la collecte de données de discours parce qu'elle veut avoir accès au vécu des personnes à partir de ce qu'elles peuvent en dire (Blanchet et Gotman, 2007).

En d'autres mots, en recherche qualitative, le chercheur «essaie de capter des données sur les perceptions d'acteurs locaux de l'intérieur» (Miles et Huberman, 2003, p. 21). En somme, à travers le discours de dirigeants, nous tenterons de comprendre ce qu'ils vivent par rapport à la diffusion d'informations financières sur l'Internet. Pour ce faire, nous avons rencontré dix dirigeants de PME du Marché Libre afin de réaliser avec eux des entretiens de recherche qualitative (Gavard-Perret et al., 2008 ; Kauffman, 1996 ; Savoie-Zajc, 2009). L'analyse qualitative des entretiens a été réalisée selon les principes de l'analyse thématique (Paillé et Mucchielli, 2008 ; Corbin et Strauss, 2008). Cette analyse ne porte pas sur le discours en tant que tel, mais bien sur le vécu que nous cherchons à comprendre.

\subsection{Analyse des sites internet}

Létude de Pozniak (2010) avait notamment pour objectif d'attribuer un score de communication financière sur l'Internet aux entreprises du Marché Libre belge. Pour ce faire, la chercheure avait construit une grille d'analyse des sites internet, basée sur les recommandations des écrits scientifiques en termes de diffusion d'informations financières sur l'Internet (voir 1.1.). La présente recherche s'inspire de cette grille afin d'appréhender l'effort volontaire de communication financière des entreprises étudiées. Dans cette grille, un point est attribué pour chaque élément d'informations financières présent sur le site internet de l'entreprise. Un score de communication financière sur l'Internet est ainsi obtenu pour chaque entreprise (voir Annexe 1). 


\subsection{Entretiens semi-directifs}

Derèze (2009, p. 107) rappelle que l'entretien est une méthodologie prééminente en sciences humaines et sociales qui est particulièrement indiquée pour " aborder des questions liées aux sens que les acteurs attribuent aux événements ou à leurs actions; aux événements eux-mêmes; aux représentations; aux valeurs ou aux pratiques». Quant à l'entretien semi-directif, il se situe entre l'entretien directif où les questions sont définies et ordonnées de la même manière pour tous les entretiens, et l'entretien non directif où l'enquêteur lance le sujet et se laisse ensuite porter à la fois par ses objectifs de recherche et par les dires de l'interviewé. En effet, lors d'entretien semi-directif, l'enquêteur s'appuie sur un guide d'entretien reprenant les thématiques à aborder sans qu'un ordre précis doive être respecté (Savoie-Zajc, 2009).

Demazière et Dubar (2007) énoncent trois postures d’analyse différentes selon la place qui est accordée à la parole des interviewés. La posture illustrative permet au chercheur de sélectionner les extraits d'entretiens qui appuient ses résultats d'analyse. La posture restitutive attribue une importance capitale aux paroles des interviewés et transmet tels quels ces propos au lecteur. La posture analytique, dans laquelle nous nous positionnons, vise la production de sens.

En ce qui concerne notre recherche, l'entretien a débuté par cette question : «À quoi sert l'Internet dans votre entreprise ? » afin de laisser toute latitude aux dirigeants d'exprimer leur opinion et d'essayer de cerner, dans un premier temps, ce quévoque cet outil pour eux. Le thème de la communication financière et de la relation avec les investisseurs n’a été abordé qu’ensuite.

Le logiciel libre WeftQDA a été utilisé lors de la phase d'analyse des entretiens. Ce logiciel, comme les autres logiciels d'assistance à l'analyse qualitative, permet de réaliser les analyses avec profondeur parce qu'il donne accès rapidement à toutes les données et donc qu'il facilite plusieurs lectures de l'ensemble, de même qu'une lecture méticuleuse de certains passages. Il facilite aussi une visée d’exhaustivité ou de complétude dans l'analyse thématique des données à cause, notamment, de ses fonctions de « recherche textuelle».

\subsection{Corpus}

Notre corpus a été construit en trois temps. Durant l'année académique 2009-2010, cinq dirigeants, contactés par téléphone, ont accepté de nous rencontrer. Début 2011, cinq autres dirigeants ont été sollicités par téléphone. Trois d’entre eux ont accepté un entretien, tandis que deux ont refusé. En février 2012, d’autres entretiens ont été menés. Parmi les cinq dirigeants contactés par courriel, deux ont été rencontrés. Ces deux entretiens ont démontré que la saturation était atteinte. En effet, la saturation théorique est atteinte lorsque les données collectées n'apportent rien de neuf et ne modifie pas de manière significative la théorie construite (Plouffe et Guillemette, 2012). Les entretiens ont duré, en moyenne, 45 minutes.

Le choix des dix entreprises étudiées parmi les vingt-six $\mathrm{PME}^{10}$ cotées sur le Marché Libre de Bruxelles s'est opéré dans un souci de variété. En effet, nous avons observé le contenu à caractère financier des différents sites internet et avons opté pour la diversité. Nous avons

10 En date du $1^{\text {er }}$ mars 2011. 
choisi quatre entreprises dont le score était supérieur à 15 , quatre entreprises dont le score était compris entre 10 et 15 et deux entreprises dont le score était inférieur à 10. La diversité en termes de taille et de secteur a aussi été visée, notamment parce que plusieurs recherches (Bonson et Escobar, 2002 ; Debreceny, Gray et Rahman, 2002 ; Oyelere, Laswad et Fisher, 2003) font ressortir l'impact de ces deux variables sur le niveau de diffusion d'informations financières sur l'Internet.

Soulignons que le corpus de dirigeants n'est pas représentatif de tous les dirigeants du Marché Libre de Bruxelles, mais cela ne constitue pas une limite à notre recherche. La représentativité est requise lorsque l'étude a pour objectif la généralisation des résultats à toute la population à l'étude, ce qui n'est pas notre cas. L'ambition de cette recherche est de comprendre un phénomène vécu. Par ailleurs, les dirigeants rencontrés remplissent les critères de bons informateurs étant donné qu'ils vivent le phénomène que cette étude vise à comprendre et qu'ils se sont révélés capables de nous parler de ce phénomène.

Notre corpus d’entreprises et ses caractéristiques sont présentés dans le tableau 2 ci-dessous.

TAbleau 2. Présentation de notre Corpus

\begin{tabular}{cclccl}
\hline $\boldsymbol{P M E}$ & \multicolumn{1}{c}{ Secteur ICB } & $\begin{array}{c}\text { Total } \\
\text { bilan } \\
\mathbf{2 0 0 9}\end{array}$ & $\begin{array}{c}\text { Chiffre } \\
\text { d'affaires } \\
\mathbf{2 0 0 9}\end{array}$ & $\begin{array}{c}\text { Effectif } \\
\text { moyen } \\
\text { (ETP) }\end{array}$ \\
\hline $\mathrm{X}$ & 2791 & Services d'appui professionnels & $42367000 €$ & $79130000 €$ & 230 \\
\hline $\mathrm{Y}$ & 9530 & Logiciels et services informatiques & $34017000 €$ & $35052000 €$ & 122 \\
\hline $\mathrm{Z}$ & 5550 & Médias & $26330300 €$ & $29252000 €$ & 245 \\
\hline $\mathrm{A}$ & 2350 & $\begin{array}{l}\text { Bâtiment et matériaux de construc- } \\
\text { tion }\end{array}$ & $10852000 €$ & $753000 €$ & 20 \\
\hline $\mathrm{B}$ & 3740 & Équipements de loisirs & $20931000 €$ & $39248000 €$ & 63 \\
\hline $\mathrm{C}$ & 4530 & Équipements et services de santé & $2315000 €$ & $3086000 €$ & 40 \\
\hline $\mathrm{D}$ & 8530 & Assurances - non-vie & $1270000 €$ & $536000 €$ & 14 \\
\hline $\mathrm{E}$ & 9530 & Logiciels et services informatiques & $1313685 €$ & $1175656 €$ & 8 \\
\hline $\mathrm{F}$ & 3570 & Agroalimentaire & $2970000 €$ & $2230000 €$ & 10 \\
\hline $\mathrm{G}$ & 8633 & Real Estate HoldingetDevelopment & $11851000 €$ & $4651000 €$ & 3 \\
\hline
\end{tabular}

Les données financières et sectorielles présentées dans ce tableau sont issues du site d'Euronext (<www.euronext.com $>$ ) et concernent l'exercice 2009 (sauf pour l'entreprise D dont le chiffre d'affaires est celui de 2007 et le total du bilan date de l'exercice 2008). Quant à l'effectif moyen, il est issu du logiciel Belfirst 2009.

Au regard de la définition européenne, les entreprises de notre étude entrent donc bien dans la catégorie des $\mathrm{PME}$. Plus précisément, les entreprises $\mathrm{B}, \mathrm{X}, \mathrm{Y}$ et $\mathrm{Z}$ sont considérées comme " moyennes ", les entreprises A, C, D, F et G sont considérées comme " petites ", tandis que l'entreprise $\mathrm{E}$ entre dans la catégorie des « microentreprises».

Le tableau 3 présente le profil des dix dirigeants rencontrés. Nous pouvons constater que la majorité d’entre eux ont fondé l'entreprise qu'ils dirigent et en détiennent une part importante du capital. 
Tableau 3. Profil des dix dirigeants rencontrés

\begin{tabular}{cclll}
\hline PME & $\hat{\text { Age }}$ & $\begin{array}{l}\text { Capital } \\
\text { détenu }\end{array}$ & Niveau de formation & Expérience dans l'entreprise \\
\hline $\mathrm{X}$ & 42 & $80 \%$ & Universitaire $(\mathrm{Bac}+5)$ & Fondateur et dirigeant \\
\hline $\mathbf{Y}$ & 47 & $16,42 \%$ & Universitaire $(\mathrm{Bac}+5)$ & Fondateur et dirigeant \\
\hline $\mathbf{Z}$ & 49 & $66 \%$ & Universitaire $(\mathrm{Bac}+5)$ & Fondateur et dirigeant \\
\hline $\mathbf{A}$ & 67 & $37 \%$ & Universitaire $(\mathrm{Bac}+5)$ & Fondateur et dirigeant \\
\hline $\mathbf{B}$ & 5 & 0 & Universitaire $(\mathrm{Bac}+5)$ & Directeur financier et administratif \\
\hline $\mathbf{C}$ & 40 & $80 \%$ & $\mathrm{Bac}+3$ & Fondateur et dirigeant \\
\hline $\mathrm{D}$ & 46 & $66 \%$ & Universitaire $(\mathrm{Bac}+5)$ & Fondateur et dirigeant \\
\hline $\mathbf{E}$ & 51 & $35 \%$ & $\mathrm{Bac}+3$ & Fondateur et dirigeant \\
\hline $\mathbf{F}$ & 47 & $17 \%$ & $\mathrm{Bac}+3$ & Cofondateur et co-dirigeant \\
\hline $\mathbf{G}$ & 49 & $60 \%$ & Universitaire $(\mathrm{Bac}+5)$ & Fondateur \\
\hline
\end{tabular}

\section{RÉSULTATS ET DISCUSSION}

Le premier point présentera les résultats de l'analyse des sites internet tandis que le second présentera les résultats des entretiens menés auprès des dirigeants. Enfin, le troisième point tentera détablir un lien entre les deux premiers.

\subsection{Résultats de l'analyse des sites internet}

Tout d'abord, soulignons que les dix entreprises de notre échantillon présentent toutes un site vitrine, c'est-à-dire « une présentation numérique de l'entreprise qui permet d’être présent sur le net comme une carte ou pressbook numérique " (<dicodunet.com $>$ ). Aucun des sites n'est dit « marchand » ce qui signifie que les clients des dix PME ne peuvent acheter les produits de l'entreprise en ligne. Parmi les quatre phases d'évolution du commerce électronique définies par Vescovi et Iseppon (2002), nous constatons que les dix entreprises se trouvent dans la première phase, appelée présence institutionnelle, où le site internet est un simple outil de présentation de l'entreprise; il fournit des informations générales et est très peu interactif.

Cette section s'intéresse essentiellement à l'information financière disponible sur le site internet des entreprises étudiées. Un tableau présentant les résultats de l'analyse des sites internet est disponible en annexe.

Neuf entreprises de notre échantillon présentent un espace dédié aux investisseurs sur leur site internet, comme le recommandent les écrits spécialisés sur la gestion communicationnelle (Khadaroo, 2005 ; Pervan, 2006 ; Barredy et Darras, 2008). Toutefois, les autres éléments identifiés par les écrits comme intéressants pour les investisseurs ne font pas l'unanimité auprès des dix dirigeants interviewés. Sept PME diffusent leur prospectus d'introduction (Euronext, 2006 ; Léger, 2008), huit présentent une revue de presse (Allam et Lymer, 2003 ; Gowthorpe, 2004 ; Khadaroo, 2005 ; Pervan, 2006 ; Euronext, 2006 ; Dutta et Bose, 2007 ; Léger, 2008) et proposent un lien vers le site d'Euronext afin de suivre le cours de leur titre. 
L'historique du cours de Bourse est présenté par deux entreprises. Toutefois, aucune névoque les dividendes distribués (Allam et Lymer, 2003 ; Xiao, Yangb et Chow, 2004 ; Khadaroo, 2005 ; Lybaert, 2005 ; Pervan, 2006 ; Dutta et Bose, 2007 ; Léger, 2008 ; Barredy et Darras, 2008 ; Gabteni, 2011). Les entreprises X, Y et Z présentent leurs rapports annuels sur plusieurs années ainsi que les chiffres clés. Outre les rapports annuels et chiffres clés, l'entreprise A fournit l'ensemble des documents comptables et financiers reconnus comme incontournables par les écrits spécialisés tels que le rapport d’audit (Lybaert, 2005 ; Dutta et Bose, 2007), le rapport du commissaire aux comptes, le procès-verbal de l'assemblée générale et le rapport du conseil d'administration (Pervan, 2006 ; Euronext, 2006 ; Dutta et Bose, 2007 ; Barredy et Darras, 2008 ; Léger, 2008). L'entreprise G met son rapport d'audit et son rapport de gestion à disposition sur son site internet. L'entreprise C présente ses rapports annuels de 2006 à 2008. L'entreprise B fournit simplement les chiffres clés de son activité tel que le recommande Euronext (2006). Les seules informations à caractère financier diffusées par l'entreprise D sont les chiffres clés datant de 2006 ; tandis que les entreprises $E$ et $F$ ne donnent aucune information financière chiffrée. Les entreprises $A, B, F, X, Y$ et $Z$ dédient un contact spécifique aux investisseurs ; les entreprises $\mathrm{B}, \mathrm{X}, \mathrm{Y}$ et $\mathrm{Z}$ diffusent le calendrier des actionnaires.

Les entreprises $D, G, X, Y$ et $Z$ présentent leur équipe dirigeante. La structure de leur actionnariat est mise en avant par les entreprises D, X et Y (Euronext 2006 ; Dutta et Bose, 2007 ; Léger, 2008 ; Barredy et Darras, 2008).

Bref, toutes les prescriptions en matière de communication financière sur l'Internet, issues des écrits scientifiques, sont loin dêtre unanimement respectées. Toutefois, nous pouvons constater qu'un certain effort volontaire de communication est réalisé par ces PME. En effet, rappelons qu'aucune communication financière n'est exigée sur le Marché Libre. Toute communication émanant des entreprises est donc volontaire selon la définition de Pourtier (2004).

\subsection{Résultats des entretiens}

\subsubsection{INTERNET C'EST AVANT TOUT LE SITE INTERNET}

Nous débutons chaque entretien par cette question : «À quoi sert l'Internet dans votre entreprise?». Les dirigeants rencontrés parlent alors de leur site internet et de son rôle d'outil de communication :

C'est un support. Notre site sert à faire connaître (entreprise C).

Le site internet, c'est la carte de visite de l'entreprise (entreprise D).

L'Internet évoque plusieurs choses au dirigeant de l'entreprise A. Il parle des courriels, des offres de prix que ces derniers permettent d'envoyer, de Skype qui permet le travail à domicile et la réduction du coût des communications, et enfin du site internet.

Deux entreprises ( $\mathrm{B}$ et $\mathrm{F}$ ) ont une utilisation plus poussée de leur site internet. Dans l'entreprise F, l'Internet est un outil de travail au quotidien. En effet, les distributeurs remettent leur rapport sur le serveur de l'entreprise et engendrent ainsi les commandes. L'entreprise B propose un outil de visualisation des stocks en ligne, à destination de ses distributeurs. Bien quaucun achat en ligne ne soit possible, ces distributeurs peuvent, à l'aide de leur login, par- 
courir le catalogue et visualiser l'état des stocks. Par ailleurs, le site internet de l'entreprise B sert également d’outil de comptabilité analytique en interne.

Le rôle du secteur d'activités a été mis en lumière par Vescovi et Iseppon (2002) dans leur étude des facteurs déterminant le développement des activités de marketing sur l'Internet. Ils découvrent que les entreprises évoluant sur des marchés de consommation utiliseraient plutôt l'Internet comme moyen de communication. Par contre, les sociétés des secteurs business-tobusiness $(B t o B)$ sont plus encouragées à se concentrer sur des éléments de service au client et moins sur la communication. C'est le cas des entreprises $B$ et $F$ qui passent toutes les deux par des distributeurs et ont une utilisation plus poussée de leur site internet.

Pour les deux entreprises fournissant des services informatiques (E et Y), l'Internet est un métier, une évidence :

C'est notre métier. C'est vraiment notre core business. Tout le groupe est basé sur l'Internet et tourne autour de l'Internet (entreprise Y).

Pour moi l'Internet, c'est un outil de base. C'est déjà des questions que je ne me pose pas. C'est comme si on me posait comme question à quoi sert la voiture dans votre société... ça fait partie de notre vie (entreprise E).

En somme, pour plusieurs dirigeants rencontrés, l'Internet est systématiquement associé au site internet. D’autres entreprises ont un niveau supérieur d'utilisation du site internet où celui-ci sert littéralement d'outil de gestion (gestion des stocks, des ventes, de la comptabilité analytique). Ces entreprises évoluent dans le BtoB. Quant aux entreprises du secteur IT, l'Internet fait partie de leur core business, c'est l'outil de base de leur activité. Ces constatations sont représentées dans la figure 1 ci-dessous.

Figure 1. Vision DE L'INTERNET

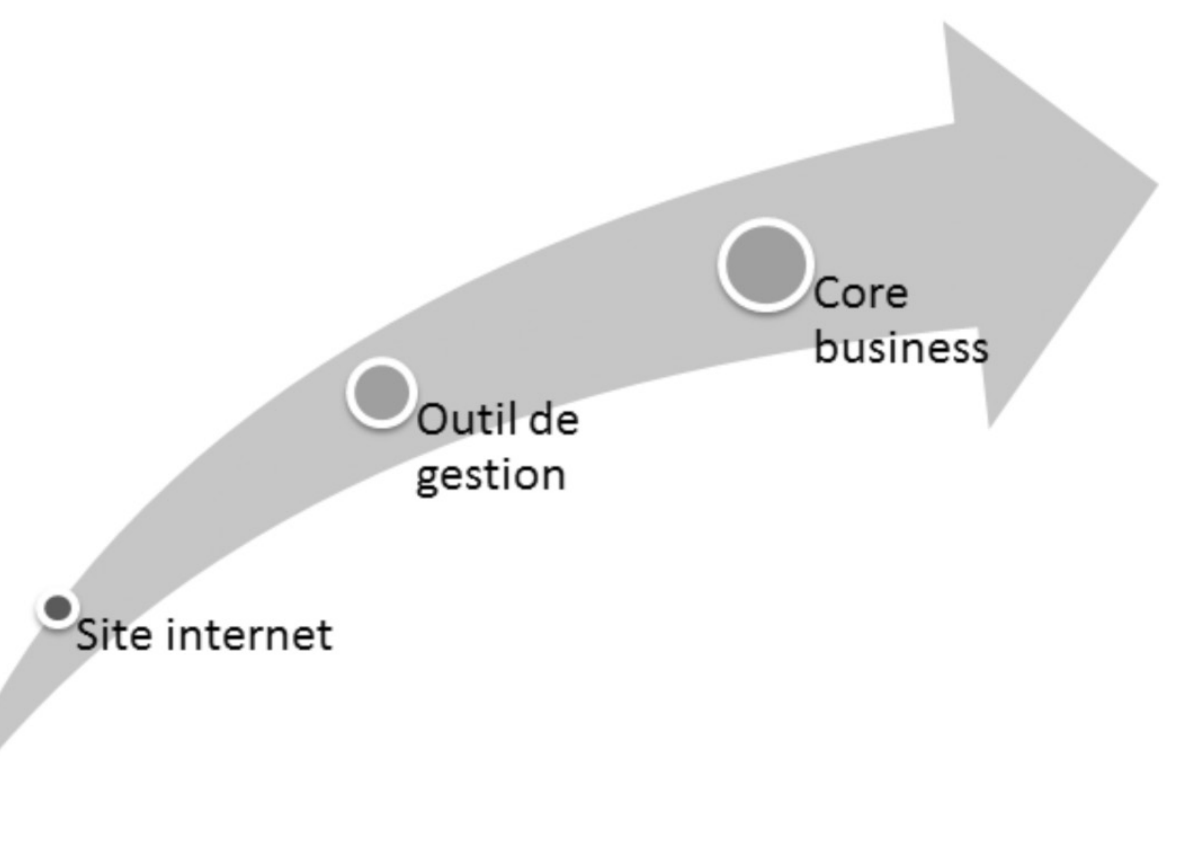




\subsubsection{COMMUNICATION FINANCIÈRE : ENTRE MÉFIANCE ET RESPECT}

Les dirigeants rencontrés ne font pas de la communication financière leur préoccupation première. Le premier signe relativement révélateur que nous pouvons mettre en avant est le fait que ce thème n'est abordé par aucun dirigeant spontanément. C’est systématiquement l'interviewer qui aborde ce sujet. Une fois abordée, la thématique de la communication financière ne fait pas du tout l'unanimité.

Un certain nombre de difficultés liées à la communication financière sont évoquées, ainsi que des raisons pouvant justifier la présence ou l'absence d'informations à caractère financier sur le site internet des entreprises.

\subsubsection{1. Éléments défavorables à la communication financière sur le net}

Les dirigeants de PME vivent certains éléments comme des freins à leur communication financière sur l'Internet. Trois dirigeants (entreprises E, F et G) affirment s'inquiéter de la diffusion d'information à la concurrence :

Pour vivre heureux, il vaut mieux vivre caché... Au niveau des chiffres, il vaut mieux faire pitié qu'envie (entreprise F).

De par notre métier, on ne peut pas trop dire certaines choses (entreprise $G$ ).

Certaines réserves sont émises au sujet de la communication financière et de la transparence :

Le fait de communiquer n'est pas nécessairement bien communiquer... Comment être transparent quand, soi-même, on ne sait pas toujours où on va (entreprise E).

Il faut faire attention à ce qu'on communique, ne pas être trop optimiste, trop pessimiste, essayer d'être juste (entreprise $G$ ).

Deux dirigeants font remarquer les moyens humains et financiers limités que peuvent consacrer les PME à leur communication financière. Ils confirment ainsi les conclusions de Raymond et al. (1990), de Sutanonpaiboon et Pearson (2006), ainsi que Westphalen et Libaert (2009).

Dans les grandes sociétés internationales cotées en Bourse, il y a un service de gens qui sont dédiés à la communication financière; donc, c'est beaucoup plus facile (entreprise F).

Par ailleurs, trois dirigeants (entreprises C, D et E) soccupent personnellement de la communication et de la gestion de leur site internet. Ceci confirme l'existence d'une proximité fonctionnelle (Torrès, 2007a) selon laquelle le dirigeant est polyvalent et assure généralement plusieurs fonctions. Cela fait également référence à l'une des caractéristiques définissant la PME selon Julien (1997), à savoir : la centralisation de la gestion autour du dirigeant.

Soulignons que ces entreprises sont considérées comme «petites » ou « micro » selon la définition européenne. Dans les autres PME (entreprises A, B, F, G, X, Y et Z), le dirigeant est épaulé pour gérer le site internet de l'entreprise, soit par la personne responsable de l'informatique (entreprises A et X), soit par l'assistante de direction (entreprises F et Z), soit par l'adjoint marketing (entreprise Y), soit par le département graphique (entreprise B), soit par un collaborateur (entreprise G). Pour ces personnes ressources, la gestion du site internet 
n'est qu'une tâche parmi d’autres. Comme le définit Julien (1997), nous pouvons observer la faible spécialisation du personnel dans la répartition des tâches ainsi que le manque de personne spécialisée dans les technologies de l'information identifié par Sutanonpaiboon et Pearson (2006). Aucune entreprise ne dispose d'une personne à temps plein chargée de gérer le site internet de l'entreprise. Ceci corrobore les constatations de Westphalen et Libaert (2009, p. 67) : "Chez les PME, les directions de la communication sont moins fréquentes ».

Certains dirigeants ont d'autres priorités que l'information des investisseurs. Trois dirigeants (entreprises B, D et E) ont comme priorité les affaires :

Je garde mon attention sur le business (entreprise B).

La priorité, c'est de faire du chiffre d'affaires... on est en manque de transparence parce qu'on est dans notre guidon (entreprise D).

Le number one, c'est de faire du business... c'est préserver le capital, mon capital, le capital de mon frère et le capital des actionnaires (entreprise E).

En effet, les dirigeants de PME ont des priorités qui divergent de celles des grandes entreprises. À cet égard, citons la recherche de St-Pierre (1999) qui souligne que, contrairement au gestionnaire d'une grande société qui a «pour responsabilité de maximiser la valeur du patrimoine des actionnaires ", l'entrepreneur ne poursuit pas cet objectif de maximisation de la valeur marchande de l'entreprise. L'entrepreneur, parce qu'il a "investi une partie importante de son capital financier et humain dans l'entreprise... », poursuit des objectifs divers tels que l'autonomie, la satisfaction, l'indépendance. De plus, Cooley et Edwards (1983) démontrent que la maximisation du revenu net de l'entreprise est le premier objectif évoqué par les dirigeants, suivi par la maximisation de la croissance du revenu net. La maximisation de la valeur marchande n'apparaissait qu'en sixième position.

Le Marché Libre et ses particularités semblent également être mis en cause par certains dirigeants rencontrés. En outre, les dirigeants rappellent qu'une très faible partie de leur capital est aux mains d'actionnaires particuliers (free float entre 3 et $20 \%$ ). Ceci rappelle le principe mis en avant par Torres (2007a) selon lequel il existerait une finance de proximité dans la PME : le capital de l'entreprise est peu dispersé dans le public, le dirigeant conserve la majorité des titres. Dans ce contexte, Labelle et Schatt (2005) avancent que la communication financière permet d'accroître les volumes de transactions, de susciter l'intérêt des analystes et donc d'améliorer la liquidité des titres de l'entreprise à capital concentré. Les dirigeants rencontrés ne partagent pas cet avis. Bien au contraire, ils déplorent le manque de liquidité des titres et l'absence de réaction de ce marché, peu importe leurs efforts de communication :

Le marché n'est pas liquide, ils ne sont pas contents, quand ils veulent vendre il faut 15 jours (entreprise $Y$ ).

J'ai dû me rendre à l'évidence, quoique je dise dans la presse, le cours ne réagit pas (entreprise B).

D’autres dirigeants regrettent le manque de couverture médiatique et le peu d'intérêt témoigné par l'État et les autorités de contrôle.

Il ressort donc que les dirigeants rencontrés perçoivent certains éléments comme des entraves à la communication financière sur l'Internet, ceux-ci sont représentés dans la figure 2 . 
FiguRE 2. ÉLÉMENTS DÉFAVORABLES À LA COMMUNICATION FINANCIÈRE SUR L'INTERNET.

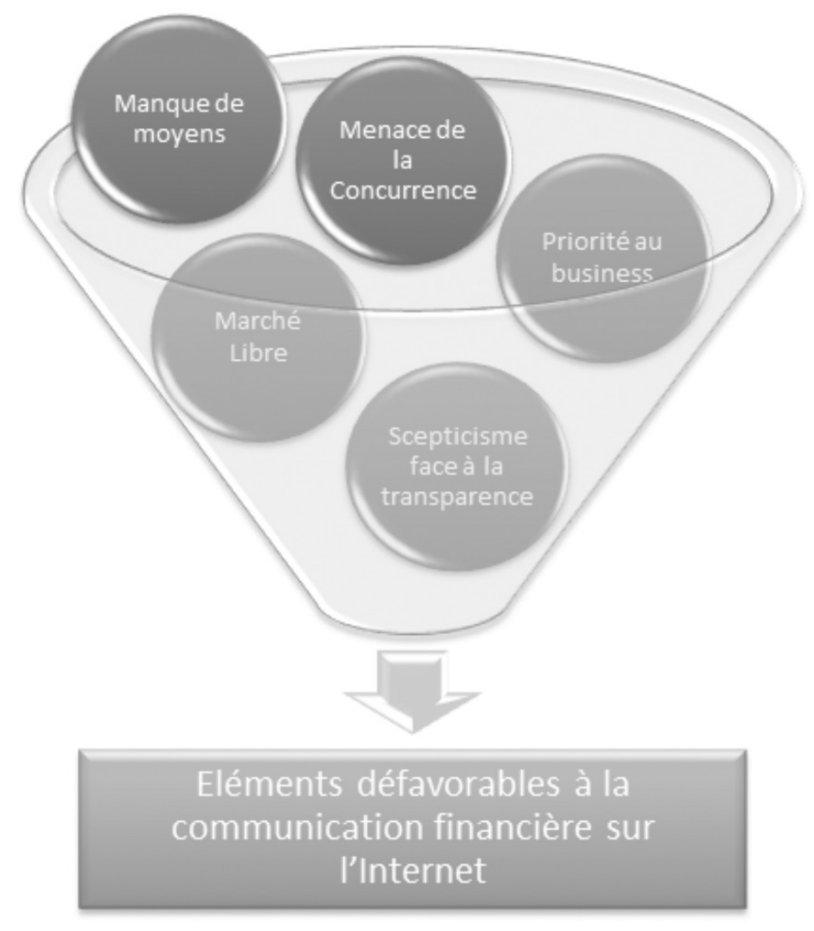

La présence de l'un ou l'autre de ces éléments n’implique pas forcément que l'entreprise ne communiquera pas d'information financière sur son site internet. Cela veut simplement dire que les dirigeants rencontrés ont identifié ces éléments comme défavorables à la communication financière en ligne.

\subsubsection{2. Éléments favorables à la communication financière sur le net}

Malgré les lacunes évoquées précédemment, certains dirigeants mettent un point d'honneur à communiquer des informations financières à travers leur site internet.

Le dirigeant de l'entreprise E semble accorder de l'importance à la nécessité d'assurer la visibilité de l'entreprise :

C'est hyper important de montrer que la société vit, fait des choses, qu'il y a de la stratégie, même si ça va moins bien, quon continue ses efforts (entreprise E).

Les dirigeants $(\mathrm{B}, \mathrm{C}, \mathrm{X}, \mathrm{Y}$ et $\mathrm{Z})$ diffusent des informations à destination de leurs investisseurs par respect :

C'est une question de principe, on est sur le marché libre, on assume (entreprise Y).

C'est une façon d'être "fair » vis-à-vis des gens qui nous ont fait ou nous font confiance (entreprise Z).

La communication que je fais, c'est juste par correction (entreprise B). 
Un actionnaire, qu'il soit sur le Marché Libre ou ailleurs, il a le droit de savoir. Il doit être respecté (entreprise C).

Les découvertes de Westphalen et Libaert (2009, p. 31), selon lesquelles l'actionnaire tendrait à devenir " la cible principale de la communication d'entreprise », ne se vérifient pas pour notre corpus. De même, ne fait pas l'unanimité, leur théorie selon laquelle (2009, p. 331) « la seule bonne stratégie possible est une meilleure transparence, la visibilité, un dialogue nourri avec le marché, le respect des règles déontologiques claires donnant la même information à tout le monde. Bref, il faut être bon élève, dire la vérité est payant. En travaillant la transparence, on établit le capital confiance de l'entreprise qui introduit une vision dynamique et favorise la tenue de son cours en toutes circonstances ». Toutefois, on peut souligner un réel effort de communication à destination des investisseurs par ces dirigeants qui respectent leurs actionnaires, mêmes minoritaires, et qui jouent le jeu des entreprises cotées.

À travers les entretiens réalisés, il apparaît que deux éléments sont propices à la diffusion d'informations financières sur le site internet des PME.

FIGURE 3. ÉLÉMENTS FAVORABLES À COMMUNICATION FINANCIÈRE SUR L'INTERNET.

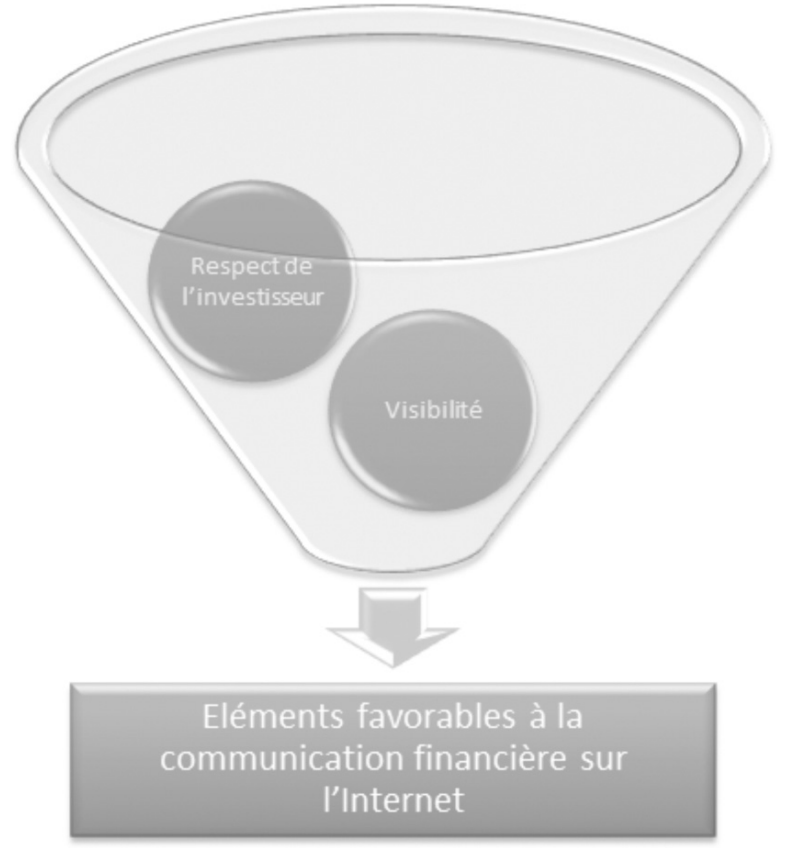

Lien entre les informations financières diffusées sur le site internet et les raisons évoquées par les dirigeants

À la lumière des propos recueillis, nous constatons que plusieurs éléments entrent en ligne de compte dans le choix du dirigeant de diffuser ou non des informations financières sur le site internet de sa PME. Ces éléments sont schématisés sous la forme d’une balance (voir Figure 4), certains ayant un poids en faveur de la communication financière sur le net, d'autres ayant un poids en sa défaveur. 
Figure 4. SYNTHÈSE COMMUNiCATION FINANCIÈre SUR L'INTERNET

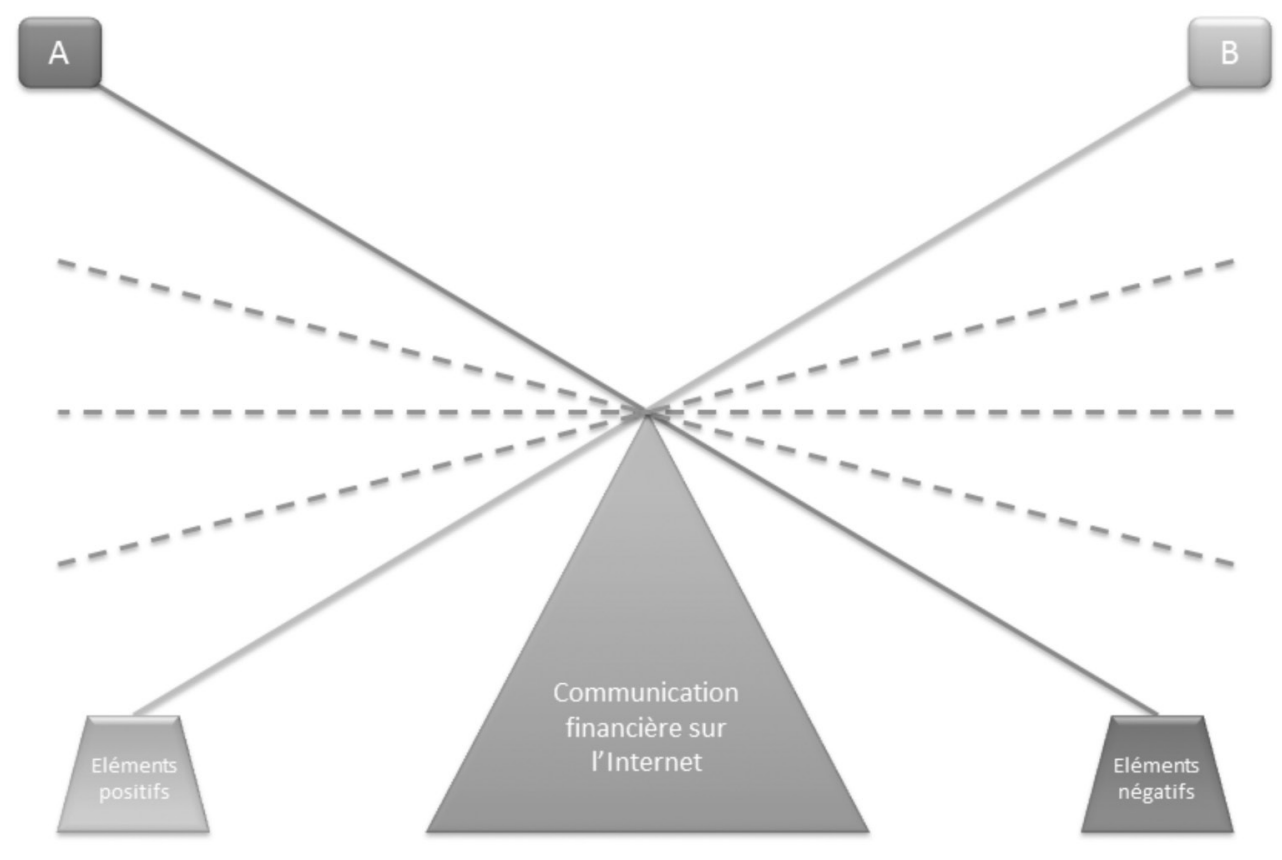

Dans le cas de figure représentée par l'axe A, les éléments négatifs ont plus de poids que les éléments positifs. La balance penche alors en défaveur de la diffusion d'informations financières sur le site internet de l'entreprise

À l'inverse, dans le cas de figure représentée par l'axe B, les éléments positifs ont plus de poids que les éléments négatifs. La balance penche alors en faveur de la diffusion d'informations financières sur le site internet de l'entreprise.

Entre ces deux cas de figure se trouvent plusieurs possibilités de «balance » de communication, représentées par les axes en pointillé.

Lorsque l'on confronte les scores de communication financière sur Internet (voir Annexe 1) et les éléments favorables et défavorables mis en avant par les dirigeants, il semblerait que certains éléments aient plus de poids que d'autres.

Tout d'abord, il apparaît que ce n'est pas leur vision d'Internet qui ait le plus de poids dans la balance. En effet, ce ne sont pas les dirigeants pour qui le site internet est le plus important qui communiquent le plus d'informations financières sur leur site internet. Les entreprises $\mathrm{E}$, Y, Z font partie du secteur IT et l'Internet fait partie de leur core business, comme expliqué au point 3.2.1. (voir Figure 1). Or, elles ne présentent pas la même quantité d'informations financières sur leur site internet et nobtiennent donc pas le même score $(E=5, Y=16, Z=15)$. Le dirigeant de l'entreprise $\mathrm{E}$ justifie sa réticence à communiquer des informations financières par sa crainte des concurrents. 
Les entreprises $\mathrm{B}$ et $\mathrm{F}$, toutes deux dans le Business to Business, partagent la même vision d'Internet. Elles n'ont toutefois pas le même score $(B=10$ et $F=4)$. Ce qui amène le dirigeant de l'entreprise $\mathrm{B}$ à communiquer davantage, c'est son souci de respecter ses investisseurs.

Ensuite, il semblerait que leur appréciation du Marché Libre, bonne ou mauvaise, ne soit pas l'élément décisif à la décision de communiquer ou pas des informations financières sur le site internet de l'entreprise. En effet, les dirigeants ( $\mathrm{A}, \mathrm{X}, \mathrm{Y}$ et $\mathrm{Z})$ les plus critiques à l'égard du fonctionnement du Marché Libre sont ceux présentant les meilleurs scores et donc diffusent toute une série d'informations à caractère financier sur le site internet de l'entreprise. A contrario, le dirigeant (C) ravi de sa cotation, n’est pas celui qui présente le score le plus élevé. Quant à ceux qui reconnaissent certains avantages à leur cotation sur ce marché, ils présentent les scores les plus faibles.

Aucun lien avec le profil des dirigeants (voir Tableau 3) ne peut être établi. Soulignons à cet égard la relative homogénéité de ces profils et la difficulté de cerner l'implication de ces différences minimes. En effet, ces dirigeants ont, en moyenne, entre 40 et 50 ans, un diplôme détudes supérieures (universitaire : 7/10, non universitaire : 3/10) détiennent une partie importante du capital de l'entreprise qu'ils ont fondée et occupent le poste de dirigeant.

De plus, aucun lien ne peut être établi entre la structure de propriété des entreprises de notre corpus et le niveau de communication financière. Les entreprises $\mathrm{Y}, \mathrm{B}$ et F, présentant un actionnariat très dilué, devraient selon Labelle et Schatt (2005) diffuser davantage d'informations financières. Or, les entreprises $\mathrm{B}$ et $\mathrm{F}$ communiquent peu déléments à caractère financier sur leur site internet (voir Annexe).

En somme, il semblerait que l'élément ayant le plus de poids dans la balance, du côté positif, soit la volonté du dirigeant de respecter ses investisseurs. En effet, les PME présentant le plus d'informations à caractère financier sur leur site internet sont celles dont le dirigeant dit vouloir assumer la cotation de sa PME sur un marché boursier et communiquer vers ses investisseurs, mêmes minoritaires, par courtoisie et respect.

Lélément faisant le plus pencher la balance du côté de la non-communication semble être la méfiance du dirigeant à l'égard de ses concurrents. En effet, les entreprises communiquant le moins d'informations financières sur leur site internet sont celles dont le dirigeant manifeste une certaine méfiance à l'égard de la concurrence.

Un lien peut donc être établi entre les informations financières communiquées sur le site internet de l'entreprise et les raisons avancées par les dirigeants. La place prépondérante du dirigeant et l'influence de ses perceptions et motivations sur la prise de décision dans l'entreprise sont donc à mettre en lien avec les résultats de notre étude exploratoire (Raymond et al., 1990 ; Filion, 2007b ; Torres, 2007a ; St-Pierre, 1999).

\section{CONCLUSION}

Les écrits dans le domaine reconnaissent indéniablement les vertus de l'Internet : "Le site web est le point de départ, le cœur du système. Très porteur en termes d'image et d'information... " (Westphalen et Libaert, 2009, p. 167). Léger (2008, p. 17) ajoute que l'Internet « a permis la mondialisation de la communication économique, avec une unité de temps de l'ordre de la 
seconde pour des investisseurs répartis à travers le monde, défiant frontières et fuseaux horaires, des investisseurs qui ont de plus en plus accès à une information considérable, dans des conditions d'égalité d’accès formidablement améliorée ».

Notre problématique nous a amenés à étudier le lien entre les données à caractère financier disponibles sur le site internet de l'entreprise et les raisons, en faveur ou non de la communication financière sur l'Internet, avancées par les dirigeants. Afin de répondre à cette question, des entretiens semi-directifs, auprès de dix dirigeants de PME cotées sur le Marché Libre de Bruxelles, ont été menés, d’une part ; d’autre part, les sites internet des entreprises rencontrées ont été analysés.

L'analyse des sites internet des dix PME a montré que toutes les recommandations, en termes de diffusion d'informations financières sur l'Internet, nétaient pas respectées ; mais qu'un réel effort de communication financière était constaté.

À la lumière des propos recueillis lors des entretiens, il s'avère que plusieurs éléments entrent en ligne de compte lors de la décision de diffusion d'informations financières sur le site internet de l'entreprise. Des éléments défavorables ont été identifiés tels que la menace de la concurrence, le scepticisme face à la transparence, le manque de moyens et la priorité au business. Certaines caractéristiques du Marché Libre telles que le manque de liquidité, de réaction du marché, de couverture médiatique, d'intérêt par l'État et les autorités de contrôle peuvent également être perçues comme des freins à la communication financière sur le site internet de l'entreprise. Quant aux éléments favorisant la divulgation d'informations financières sur le net, les dirigeants évoquent le désir de visibilité et le respect des investisseurs, même minoritaires.

Quant au lien avec le contenu du site internet, il apparaît que les dirigeants mentionnant leur crainte face à la concurrence sont ceux dont le site internet diffuse le moins d'informations financières. Tandis que les dirigeants évoquant leur volonté de respecter les investisseurs sont ceux dont le site internet présente le plus d'informations à caractère financier. Ces résultats d'une étude exploratoire fournissent un éclairage sur les différents aspects du phénomène que nous cherchions à comprendre. Ils fournissent ainsi beaucoup d'hypothèses de compréhension que nous naurions pas pu intuitionner sans cette analyse qualitative inductive des données fournies directement par les dirigeants que nous avons rencontrés.

Une des limites de notre étude réside dans la constitution du corpus. Seul l'avis des dirigeants qui ont accepté de nous rencontrer a pu être recueilli. De plus, rappelons-le, notre échantillon n’est pas représentatif parce que notre étude n’a pas de visée de généralisation.

Par contre, la compréhension que nous avons pu construire méthodiquement ouvre des avenues de recherche fort intéressantes, notamment des recherches plus approfondies sur les motivations et les objectifs des dirigeants, recherches qui permettraient détablir une typologie et de faire apparaitre un lien validé statistiquement avec la communication financière sur l'Internet.

Enfin, le Marché Libre et son fonctionnement ont été pointés du doigt, par certains dirigeants rencontrés, comme étant des éléments défavorables à la communication financière sur l'Internet. Leur perception de ce marché mériterait dêtre approfondie et confrontée à celle des autres acteurs du marché : autorités de contrôle, experts, analystes, banques d'investissement et investisseurs. 
Par ailleurs, la rencontre avec des investisseurs - internautes apporterait également un regard intéressant sur le contenu financier des sites internet et sur leurs attentes en tant que partie prenante de l'entreprise.

\section{Annexe. Analyse Des sites internet}

\begin{tabular}{|c|c|c|c|c|c|c|c|c|c|c|}
\hline \multirow{2}{*}{ Eléments financiers } & \multicolumn{10}{|c|}{ PME } \\
\hline & $\mathrm{X}$ & $\mathrm{Y}$ & $\mathrm{Z}$ & $\mathrm{A}$ & $\mathrm{B}$ & $\mathrm{C}$ & $\mathrm{D}$ & $\mathrm{E}$ & $\mathrm{F}$ & $\mathrm{G}$ \\
\hline Espace actionnaire ou investisseur & $\mathrm{X}$ & $\mathrm{x}$ & $x$ & $x$ & $x$ & $x$ & $x$ & $x$ & $\mathrm{x}$ & \\
\hline Prospectus d'introduction en Bourse & & & & $\mathrm{x}$ & $\mathrm{x}$ & $\mathrm{x}$ & $\mathrm{x}$ & $\mathrm{x}$ & & \\
\hline Revue de presse & $\mathrm{x}$ & $\mathrm{x}$ & & $\mathrm{x}$ & $\mathrm{x}$ & $\mathrm{x}$ & $\bar{x}$ & $\mathrm{x}$ & & $\mathrm{x}$ \\
\hline Communiqués de presse & $\mathrm{x}$ & $\mathrm{x}$ & & $\mathrm{x}$ & & $\mathrm{x}$ & & $\mathrm{x}$ & & $\mathrm{x}$ \\
\hline Lien vers Euronext & $\bar{x}$ & & $\mathrm{x}$ & $\mathrm{x}$ & $\bar{x}$ & $\mathrm{x}$ & $\bar{x}$ & & $\mathrm{x}$ & \\
\hline Lien vers SCF (conseiller IPO) & & & & & & & & $\mathrm{x}$ & & \\
\hline Lien vers cours de Bourse sur l'Echo & $\mathrm{x}$ & & & & & & & $\mathrm{x}$ & & \\
\hline Cours de Bourse & & $\mathrm{x}$ & & & & $\mathrm{x}$ & & & & \\
\hline Rapport de gestion 09 & & & & $\mathrm{x}$ & & & & & & $\mathrm{x}$ \\
\hline Rapport du Commissaire AG 09 & & & & $\mathrm{x}$ & & & & & & \\
\hline Rapport du Conseil Administration 09 & & & & $\mathrm{x}$ & & & & & & \\
\hline PV Assemblée Générale ordinaire 10 & & & & $\mathrm{x}$ & & & & & & \\
\hline PV Assemblée Générale extraordinaire & & & & $\mathrm{x}$ & & & & & & \\
\hline Rapport annuel 09 & $\mathrm{x}$ & $\mathrm{x}$ & $\mathrm{x}$ & $\mathrm{x}$ & & $\mathrm{x}$ & & & & \\
\hline Rapport annuel 08 & $\mathrm{x}$ & $\mathrm{x}$ & $\mathrm{x}$ & & & $\mathrm{x}$ & & & & \\
\hline Rapport annuel 07 & $\mathrm{x}$ & $\mathrm{x}$ & $\mathrm{x}$ & & & $\mathrm{x}$ & & & & \\
\hline Rapport annuel 06 & & $\mathrm{x}$ & $\mathrm{x}$ & & & $\mathrm{x}$ & & & & \\
\hline Rapport annuel 05 & & $\mathrm{x}$ & $\mathrm{x}$ & & & & & & & \\
\hline Chiffres clés 10 & & $\mathrm{x}$ & & & & & & & & \\
\hline Chiffres clés 09 & & $\mathrm{x}$ & $\mathrm{x}$ & & $\mathrm{x}$ & & & & & \\
\hline Chiffres clés 08 & $\mathrm{x}$ & $\mathrm{x}$ & $\mathrm{x}$ & & $\mathrm{x}$ & & & & & \\
\hline Chiffres clés 07 & $\mathrm{x}$ & & $\mathrm{x}$ & & $\mathrm{x}$ & & & & & \\
\hline Chiffres clés 06 & $\mathrm{x}$ & & $\mathrm{x}$ & & & & $\mathrm{x}$ & & & \\
\hline Commentaires sur les chiffres clés & & & & & $\mathrm{x}$ & & & & & \\
\hline Calendrier de l'actionnaire & $\mathrm{x}$ & $\mathrm{x}$ & $\mathrm{x}$ & & $\mathrm{x}$ & & & & & \\
\hline Contact spécial investisseurs & $\mathrm{x}$ & $\mathrm{x}$ & $\mathrm{x}$ & $\mathrm{x}$ & $\mathrm{x}$ & & & & $\mathrm{x}$ & \\
\hline Structure de l'actionnariat & & $\mathrm{x}$ & & & & & $\mathrm{x}$ & & & \\
\hline Nombre d'action & & & & & & & & & & \\
\hline Statuts de la société & & & & $\mathrm{x}$ & & & & & & \\
\hline Equipe dirigeante présentée & $\mathrm{x}$ & $\mathrm{x}$ & $\mathrm{x}$ & & & & $\mathrm{x}$ & & & \\
\hline $\begin{array}{l}\text { Composition du Conseil } \\
\text { d'Administration }\end{array}$ & & & & & & & & & $\mathrm{x}$ & \\
\hline Présentations des auditeurs & $\mathrm{x}$ & & $\mathrm{x}$ & & & & & & & \\
\hline $\begin{array}{l}\text { Nombre d'éléments financiers } \\
\text { sur le site internet }\end{array}$ & 16 & 16 & 15 & 13 & 10 & 10 & 7 & 5 & 4 & 3 \\
\hline
\end{tabular}




\section{BIBLIOGRAPHIE}

Abdelsalam O.H., Bryant S.M. et Street D.L. (2007), An examination of the comprehensiveness of corporate Internet reporting provided by London-listed companies, Journal of International Accounting Research, 6, 2, 33 p.

AmARASENA A. (2008), The Internet in the performance of small exporting firms. A developed to developing country market context, Journal of Internet Business, 5, 51-80.

Allam A. et Lymer A. (2003), Developments in Internet financial reporting : review and analysis across five developed countries, International Journal of Digital Accounting Research, 3, 6, 165-200.

Almilia L.S. (2009), Determining factors of internet financial reporting in Indonesia, Accounting et Taxation, 1, 1, 87-99.

Ashbaugh H., Johnstone K. et Warfield T. (1999), Corporate reporting on the Internet, Accounting horizons, 13, 3, 241-257.

BARREDy C. et DARras N. (2008), L'utilisation d'Internet dans la communication auprès des actionnaires minoritaires dans les entreprises familiales cotées, Journal des entreprises familiales, 1, 1, 41-68.

Ben Ali C. et Gettler-Summa M. (2006), La communication financière et la structure de propriété : le cas français, comptabilité, contrôle, audit et institutions, Conférence de Association francophone de comptabilité, Hammamet, Tunisie, $30 \mathrm{p}$.

Blanchet A. et Gotman A. (2007), L'enquête et ses méthodes : l'entretien, $2^{\mathrm{e}}$ édition, Paris, Armand Colin, 128 p.

Blumer H. (1969), Symbolic interactionism : perspective and method. Englewood Cliffs, NJ, PrenticeHall, 224 p.

Bollen L., Hassink H. et Bozic G. (2006), Measuring and explaining the quality of Internet investor relations activities : a multinational empirical analysis, International Journal of Accounting Information Systems, 7, 4, 273-298.

Bonson E. et Escobar T. (2002), A survey on voluntary disclosure on the Internet : empirical evidence from 300 european union companies, The International Journal of Digital Accounting Research, 2, 1, 27-51.

BOUCHARD Y. (2000), De la problématique au problème de recherche, dans Introduction à la recherche en éducation, T. Karsenti et L. Savoie-Zajc (dir.), Sherbrooke, Québec, CRP, 79-98.

Chaganti R. et Chaganti R. (1983), A profile of profitable and not so profitable small business, Journal of Small Business Strategy, 7, 1, 1-20.

Commission Européenne (2006), La nouvelle définition des PME. Guide de l'utilisateur et modèle de déclaration, Entreprises et Industries publications, <http:// ec.europa.eu/enterprise/policies/sme/files/ sme_definition/sme_user_guide_fr.pdf>, consulté le 14 novembre 2013.

Cooley P.L. et Edwards C.E. (1983), Financial objectives of small firms, Amercian Journal of Small Business, 8, 1, 28-31.

Corbin J. et Strauss A.L. (2008), Basics of Qualitative Research, $3^{\mathrm{e}}$ edition, Thousand Oaks, CA, Sage, $312 \mathrm{p}$.

Davidsson P. (1991), Continued entrepreneurship : ability, need and opportunity as determinants of small firm growth, Journal of Business venturing, 6, 6, 405-29. 
Debreceny R., Gray G. et Rahman A. (2002), The determinants of Internet financial reporting, Journal of Accounting and Public Policy, 21, 4-5, 371-394.

Demazière D. et Dubar C. (2007), Analyser les entretiens biographiques. L'exemple de récits d'insertion, Canada, Presses universitaires de Laval, $350 \mathrm{p}$.

Derèze G. (2009), Méthodes empiriques de recherché en communication, $1^{\text {re }}$ édition, Bruxelles, Éditions De Boeck Université, 256 p.

De Zoysa A. et Herath S.K. (2007), The impact of owners/dirigeants mentality on financial performance of SMEs in japan, Journal of Management Development, 26, 7, 652-666.

Dilthey W. (1942), Introduction à l'étude des sciences humaines, Paris, Presses universitaires de France, $513 \mathrm{p}$.

Dutta P. et Bose S. (2007), Web-based corporate reporting in Bangladesh : an exploratory study, The cost and management, 35, 6, 29-45.

EuRonext (2006), Recommandations pour un parcours boursier réussi sur le Marché Libre, <http:// www.euronext.com/fic/000/010/800/108002.pdf>, consulté le 15 décembre 2006.

European Commission (2008), SBA fact sheet Belgium, Entreprise and Industry Publications, <http:// ec.europa.eu/enterprise/policies/sme/facts-figures-analysis/performance-review/files/countriessheets/2008/belgium_en.pdf>, consulté le 14 novembre 2013.

Evrard Y., Pras B. et Roux E. (2009), Market, fondements et méthodes des recherches en marketing, $4^{\mathrm{e}}$ édition, Paris, Dunod, $703 \mathrm{p}$.

Filion L.J. (2007a), Introduction, dans Management des PME. De la création à la croissance, Paris, Pearson Education, $19 \mathrm{p}$.

Filion L.J. (2007b), Types de propriétaires-dirigeants de PME, dans Management des PME. De la création à la croissance, Paris, Pearson Education, 63-73.

Fsma(2012),Obligationsincombantauxémetteurscotéssurunmarchéréglementé,CirculaireFSMA/2012_01 du 11 janvier 2012, <http://www.fsma.be/ /media/Files/circinfo/FR/gv/info/fsma_2012_01.ashx>, consulté le 15 décembre 2013.

Gabteni H. (2011), Construction d'un score de publication volontaire entendu comme une mesure de la communication financière en période pré/post IFRS, $32^{e}$ Congrès de l'Association francophone de comptabilité : comptabilités, économie et société, Montpellier, France, 10-11 mai.

Gavard-Perret M-L, Gotteland D., Haon C. et Jolibert A. (2008), Méthodologie de la recherche: réussir son mémoire ou sa thèse en sciences de gestion, Paris, Pearson Education, $400 \mathrm{p}$.

Gowthorpe C. (2004), Asymmetrical dialogue ? Corporate financial reporting via the Internet, Corporate Communications, 9, 4, 283-293.

Grandon E.E. et Pearson J.M. (2004), Electronic commerce adoption : an empirical study of small and medium US businesses, Information et Management, 42, 1, 197-216.

Ho S. et Wong K. (2001), A study of the relationship between corporate governance structures and the extent of voluntary disclosure, Journal of International Accounting, Auditing and Taxation, 10, 2, 139156.

Husserl E. (1977), Phenomenological psychology, The Hague, Martinus Nijhoff, 200 p.

Iвrahim A.B. et Goodwin J.R. (1986), Perceived causes of success in small business, American Journal of Small Business, 2, 11, 41-50. 
Investor Relations Business (2000), New York, juin 12, 1.

InVestor Relations Business (2002), New York, janvier 14, 1.

Jensen M.C. et Meckling W.H. (1976), Theory of the firm : managerial behavior, agency cost and ownership structure, Journal of Financial Economics, 3, 4, 305-360.

Julien P.-A. (1997), Les PME : bilan et perspectives, Québec, Groupe de Recherche en économie et gestion des PME, Economica, $437 \mathrm{p}$.

Kaufmann J.-C. (1996), Leentretien compréhensif, Paris, Nathan, 127 p.

Khadaroo I. (2005), Business reporting on the Internet in Malaysia and Singapore. A comparative study, Corporate Communications, 10, 1, 58-68.

Kotey B. et Meredith G.G. (1997), Relationship among owner/dirigeant personal values and perceptions, business strategies, and enterprise performance, Journal of Small Business Management, 35, 2, 37-64.

Labelle R. et Schatt A. (2005), Structure de propriété et communication financière des entreprises françaises, Finance Contrôle Stratégie, 8, 3, 77-104.

LÉGer J.Y. (2008), La communication Financière, $2^{\mathrm{e}}$ édition, Paris, Dunod, 278 p.

Lertwongsatien C. et Wongpinunwatana N. (2003), E-commerce adoption in Thailand : an empirical study of small and medium enterprises (SMEs), Journal of Global Information Technology Management, 6, 3, 67-83.

Lybaert N. (2005), On-line financial reporting. An analysis of Dutch listed firms, The International Journal of Digital Accounting Research, 2, 4, 195-234.

Marchesnay M. (1991), La PME : une gestion spécifique ?, Économie rurale, 206, 1, 11-17.

Mehrtens J., Cragg P.B. et Mills A.M. (2001), A model of Internet adoption by SMEs, Information et Management, 39, 3, 165-176.

Mendes-DA-Silva W. et Christensen T. (2004), Determinants of voluntary disclosure of financial information on the Internet by brazilian firms, $<$ http://ssrn.com/abstract $=638082>$, consulté le 30 août 2004.

Miles M.B. et Huberman M.A. (2003), Analyse des données qualitatives, $2^{e}$ édition, Bruxelles, De Boeck, $626 \mathrm{p}$.

Mirchandani D. et Motwani J. (2001), Understanding small business electronic commerce adoption : an empirical analysis, Journal of Computer Information Systems, 41, 3, 70-73.

Oyelere P., Laswad F. et Fisher R. (2003), Determinants of Internet financial reporting by New Zealand companies, Journal of International Financial Management and Accounting, 14, 1, $26-63$.

Oyelere P. et Mohamed E. (2007), Internet financial reporting in Oman, Global Journal of Business Research, 1, 2, 45-54.

PAILlÉ P. (2007), La recherche qualitative. Une méthodologie de la proximité, dans Problèmes sociaux, théories et méthodologies de la recherche, H. Dorvil (dir.), Québec, Presses de l'Université du Québec, 409-443.

Paillé P. et Mucchielli A. (2008), L’analyse qualitative en sciences humaines et sociales, $2^{\mathrm{e}}$ édition, Paris, Armand Colin, $424 \mathrm{p}$.

Pearson J.M. et Grandon E.E. (2005), An empirical study of factors that influence e-commerce adop- 
tion/non-adoption in small and medium sized businesses, Journal of Internet Commerce, 4, 4, 1-21.

Pervan I. (2006), Voluntary financial reporting on the Internet. Analysis of the practice of croatian and slovene listed joint stock companies, Financial theory and practice, 30, 1, 1-27.

Plouffe M.-J. et Guillemette F. (2012), La MTE comme apport au développement de la recherche en arts, dans Méthodologie de la théorisation enracinée (grounded theory) : riche diversité, J. Luckerhoff, et $\mathrm{F}$. Guillemette (dir.), Québec, Presses de l'Université du Québec, 302 p.

Pourtier F. (2004), La publication d'informations financières volontaires : un essai de synthèse, Comptabilité - Contrôle - Audit, 10, 1, 79-102.

Pozniak L. (2010), Financial Communication on the Web - Evidence from Belgium, Accounting et Taxation, 1, 2, 47-58.

Raymond L., Bergeron F., Gingras L. et Rivard S. (1990), Problématique de l'informatisation des PME, TIS, 3, 1, 131-148.

ST-PIERRE J. (1999), La gestion financière des PME. Théories et pratiques, PME et entrepreneuriat, Québec, Presses de l'Université du Québec, 322 p.

SAvoie-ZajC L. (2009), L’entrevue semi-dirigée, dans Recherche sociale : de la problématique à la collecte de données, 5e édition, B. Gauthier (dir.), Québec, Presses de l'Université du Québec, 337-360.

Schutz A. (1987), Le chercheur et le quotidien. Phénoménologie des sciences sociales, Paris, Méridiens Klincksieck, $286 \mathrm{p}$.

Sutanonpaiboon J. et Pearson A.M. (2006), E-commerce adoption : perceptions of dirigeants/owners of small and medium-sized enterprises (SME) in Thailand, Journal of Internet Commerce, 5, 3, 53-82.

Torres O. (2007a), Approche descriptive de la spécificité de gestion des PME : le mix de proximité, dans Management des PME. De la création à la croissance, Paris, Pearson Education, 23-33.

Torres O. (2007b), Approche explicative du comportement caractéristique des PME : le principe proxémique, dans Management des PME. De la création à la croissance, Paris, Pearson Education, 35-45.

Vescovi T. (2000), Internet communication : the italian SME case, Corporate Communications : An International Journal, 5, 2, 107-112.

Vescovi T. et Iseppon M. (2002), L'évolution d'Internet comme moyen de communication et de marketing des PMI, Revue Française du Marketing, 189, avril-mai, 131-40.

Villani A. (1996), Méthode et théorie dans l'oeuvre de Gilles Deleuze, Les temps modernes, 51, 586, $142-154$.

Weber M. (1949), The methodology of the social sciences, New York, Free Press, 188 p.

Werner A. (2008), The influence of Christian identity on SME owner-dirigeants' conceptualisations of business practice, Journal of Business Ethics, 82, 2, 449-462.

Westphalen M.-H. et Libaert T. (2009), Communicator. Toute la communication d'entreprise, $5^{\mathrm{e}}$ édition, Paris, Dunod, 493 p.

Wijewardena H. et Cooray S. (1996), Factors contributing to the growth of small manufacturing firms : perceptions of japanese owner/dirigeants, Journal of Enterprising Culture, 4, 4, 351-61.

Wong P. (2003), Global and national factors affecting e-commerce diffusion in Singapore, The Information Society, 19, 1, 19-32. 
XIAO J., YANGB H. et Chow C. (2004), The determinants and characteristics of voluntary Internet-based disclosures by listed chinese companies, Journal of Accounting and Public Policy, 23, 3, 191- 225.

\section{Webographie}

$<$ http://www.Internetworldstats.com/stats.htm>.

$<\mathrm{http}: / /$ www.insolus.com/article-internet-majuscule-minuscule\#axzz2GBOFbDWk> . 